\title{
Utility of magnetic resonance proton density fat fraction technique in quantification of liver fat in living donors for liver transplantation
}

\author{
Fatma Mohamed Sherif ${ }^{* *}$, Sabry Alameldeen Elmogy ${ }^{1}$, Rihame Mohamed Abd EL-wahab ${ }^{1}$ and
} Mohamed Abdel Wahab²

\begin{abstract}
Background: Hepatic steatosis in living donors for liver transplantation causes morbidity of both donor and recipient. This study aims at evaluating magnetic resonance proton density fat fraction technique (MR PDFF) in quantitative evaluation of living donor's hepatic steatosis compared to histopathology.
\end{abstract}

Results: The examined potential living liver donors' liver biopsies revealed hepatic steatosis $<5 \%$ (grade 0 ) in 40 donors and $5-10 \%$ (grade 1) in 7 donors. MR PDFF technique with IDEAL sequence showed excellent results for prediction and quantitative evaluation of liver fat with sensitivity, specificity, and accuracy of $85.7 \%, 97.5 \%$, and $95.7 \%$, respectively, compared to histopathology (95\% confidence interval 0.98-1.01). There was an excellent inter-reader agreement between both readers in estimation of MR liver fat fraction ( $r=0.969$ at 95\% confidence interval $0.946-0.983$ ).

Conclusion: Noninvasive hepatic MR PDFF technique with IDEAL sequence is a precise reliable technique for pre-operative quantitative assessment of hepatic steatosis in potential living liver donors.

Keywords: Hepatic steatosis, Magnetic resonance proton density fat fraction technique (MR PDFF), Living donors, Liver transplantation

\section{Background}

Living donor liver transplantation has been widely accepted as a routine treatment modality for end-stage liver disease for about 2 decades. Donor's safety is an important ethical concern, because donors are liable to many surgical and health risks [1].

Hepatic steatosis is characterized by deposition of lipid droplets in hepatocytes [2]. The prevalence was estimated to be $32 \%$ in the Middle East and 25\% globally (worldwide) [3]. It is a remarkable risk factor in hepatic surgery, and its incidence is nearly $25 \%$ in living liver donors. Many authors stated that $\geq 30 \%$ hepatic steatosis is a serious risk factor with resultant poor graft function. Even mild steatosis interferes with the recovery of the

\footnotetext{
* Correspondence: fatema_sherif@yahoo.com

${ }^{1}$ Radiology Department, Faculty of Medicine, Mansoura University, Mansoura, Egypt

Full list of author information is available at the end of the article
}

donor's remaining liver $[4,5]$. Therefore, some medical centers consider $10 \%$ steatosis as a safe cutoff value for living donor liver transplantation to conserve donor's liver. Thus, prompt quantitative evaluation of liver steatosis in potential living liver donors is of a paramount importance for donor selection [6].

Hepatic histopathological assessment is still considered the reference standard in numerous centers of liver transplantation. However, it has many drawbacks, including small sample volumes (sampling error) and inter-observer variability [7]. Moreover, it has many provisional complications like bleeding and infection, and bile leakage is relatively of low incidence in living donors [8], so, it is better to avoid this invasive procedure with availability of reliable noninvasive technique for detection of hepatic steatosis. Nowadays, in many medical centers, liver biopsy is used selectively for hepatic 
steatosis measurement if it was diagnosed by noninvasive dependable imaging modality [5].

Many noninvasive radiological studies have been increasingly used for evaluation of hepatic steatosis. Ultrasound (US) is a commonly used technique. However, it provides only qualitative assessment by evaluation of liver texture and echogenicity [8]. Computed tomography is more accurate than ultrasound, but it has its own disadvantages, including interference due to iron deposition, fibrosis, and risk of radiation exposure and lower sensitivity than MRI [9].

In some medical centers, MRI is currently used in evaluation of hepatic steatosis, due to its availability and high sensitivity for fat detection and measurement. But, evidence-based studies are limited. Available information declares how correct performance of MRI is important to overcome confounders that interfere with accuracy of conventional MRI $[2,10]$.

Many MR techniques are available for assessment of hepatic steatosis, such as dual and triple-echo gradientecho MRI, chemical shift imaging, and hydrogen $1(1 \mathrm{H})$ MR spectroscopy. In this study, we discussed MR proton density fat fraction (MR PDFF) technique, a newly available technique for measurement of hepatic steatosis. It is characterized by its short acquisition time and simple post-processing [5, 11]. To our knowledge, a limited number of studies discussed this technique. This study aimed at evaluating MRI-PDFF technique in quantitative assessment of hepatic steatosis in potential living donors for liver transplantation compared to liver biopsy.

\section{Methods}

\section{Study design and population}

This was a retrospective study, approved by our institutional ethics committee. It was conducted from August 2016, including 47 potential living liver donors for liver transplantation (37 males and 10 females). Their ages ranged from 19.5 to 37 years with the mean age $\pm \mathrm{SD}=27.01 \pm 4.9$ years old. Demographic and clinical criteria of studied group were demonstrated in Table 1.

Exclusion criteria included the existence of contraindications to MRI including claustrophobia and proof of presence of respiratory and motion artifacts in MR images, which evaluated as post-MRI exclusion criteria to prevent bias in MR PDFF assessment [12, 13].

\section{MRI acquisition}

All MR PDFF examinations were done. Liver MRI was performed with subjects scanned in supine position on a 1.5T MRI scanner (Explorer, GE Medical Systems, Milwaukee, WI, USA) using an 8-channel phased array receiver coil. A multiecho three-dimensional (3D) IDEAL sequence with fly-back gradients was employed for the evaluation of the liver steatosis (IDEAL IQ, GE
Table 1 Demographic and clinical criteria of studied donors

\begin{tabular}{lll}
\hline & $n=47$ & Percent \\
\hline Age/years & & \\
$\quad$ Mean \pm SD (MIN-MAX) & $27.01 \pm 4.9(19.5-37.0)$ & \\
Gender & & 78.7 \\
$\quad$ Male & 37 & 21.3 \\
$\quad$ Female & 10 & \\
BMI (kg/m²) & \\
Mean \pm SD (MIN-MAX) & $25.16 \pm 0.41(17.9-33.5)$ & \\
Liver weight/kg & & \\
Mean \pm SD (MIN-MAX) & $1.56 \pm 0.22(1.11-2.14)$ & \\
\hline
\end{tabular}

$S D$ standard deviation, $B M I$ body mass index, $\mathrm{kg} / \mathrm{m}^{2}$ kilogram per square meter, MIN-MAX minimum-maximum

Healthcare). The IDEAL IQ sequence is a T1independent, T2*-corrected estimating $\mathrm{R} 2 *\left(1 / \mathrm{T} 2^{*}\right)$, and chemical shift-based fat-water separation method for correction of $\mathrm{T} 2 *$ effects with multi-peak fat spectral modeling creating fat fraction maps in a shorttime single breath-hold acquisition [1 14$]$.

Patients were instructed to hold their breath at the end of inspiration during MR sequence acquisition. IDEAL IQ sequence was carried out in axial plane with the following parameters: $\mathrm{TR}=10.6 \mathrm{msec}, \mathrm{TE}=5 \mathrm{msec}$, matrix size $=160$ $\times 160, \mathrm{NEX}=0.69, \mathrm{FOV}=44 \times 44 \mathrm{~cm}$, flip angle $=7^{\circ}$, slice thickness $=8 \mathrm{~mm}, 0$ inter-slice gap, $125 \mathrm{kHz}$ bandwidth, and total examination time $=14 \mathrm{~s}$. The gradient-echo (GRE) MR images were put out in DICOM format for post-processing.

\section{MR image interpretation and data analysis}

Images were analyzed by two independent experienced radiologists on advantage window (GE volume share 7), freehand circular ROIs were placed at segments 8 and 5 of the right liver lobe and segment 2 of the left liver lobe away from visible intrahepatic blood vessels and bile ducts, ranging in size from 3.4 to $5.5 \mathrm{~cm}^{2}$ (Fig. 1) [1, 5, 8]. Each ROI has mean and standard deviation value, and the mean value of these ROIs for each patient was calculated.

\section{Donor biopsy}

Histopathology was performed within 1-month time interval between MR examination and biopsy. Percutaneous tru-cut biopsies were obtained by 18 -gauge needles after local anesthesia and US guidance. Histologic fat grading of macrovesicular steatosis was reported by experienced pathologists with quantitation of the percentage of hepatocytes containing fat droplets using hematoxylin-eosin staining $[1,5]$.

\section{Statistical analysis}

The statistical analysis of data proceeded using Statistical Package for Social Science version 22 (SPSS Inc., Chicago, 


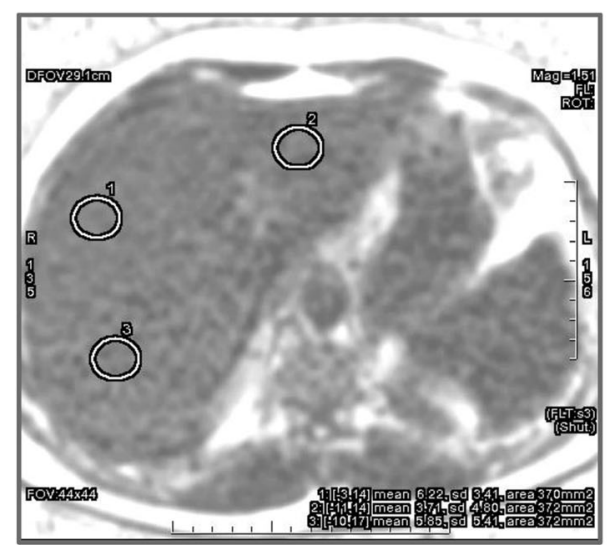

(A)

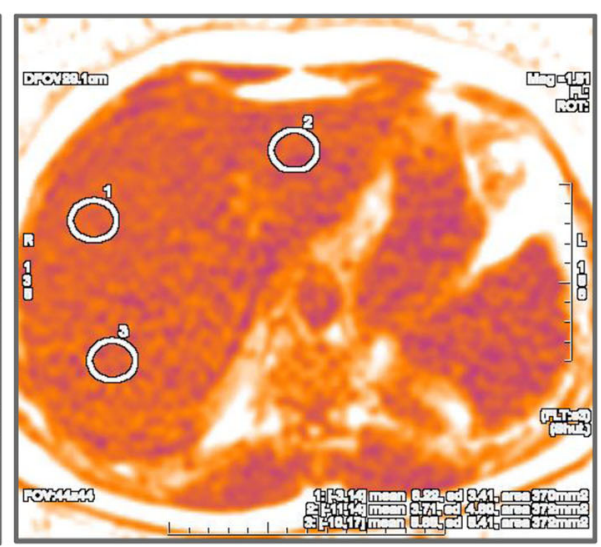

(B)

Fig. 1 a, b Representative example of ROI measurement on axial MR HFF map. Three approximately $3.4-5.4 \mathrm{~mm}^{2}$ ROls were drawn in the midplane of the liver

Ill, USA). Descriptive statistics in the form of mean, standard deviation, median, minimum, and maximum were used. Whole-liver PDFF for each studied individual was defined as the mean of the PDFFs of the determined ROIs. Also, MR and pathological grading of liver fat were performed for each measurement according to available grading system in literature [14-17]. The median and ranges of MR liver PDFFs and MR liver fat grade were estimated for the first and second observers.

Using liver histopathological fat percentage and grades as reference standards, receiver operating characteristic (ROC) curve analysis was used, calculation of sensitivity, specificity, positive, and negative predictive values as well as accuracy of MR liver PDFF technique was done. Interclass correlation was carried out to detect interreader agreement of both observers.

\section{Results}

A total 47 potential living donors for liver transplantation were studied, their demographic and clinical information were summarized descriptively (Table 1 ).

The median MR degree of macro-vesicular steatosis for the first observer was $2.5 \%$ ranging from 1.1 to $16 \%$ and for the second observer was $3.26 \%$ ranging from 1.43 to $14.7 \%$. MR liver fat grade 0 were 43 donors for the first observer and 42 donors for the second observer, while MR liver fat grade 1 were 4 donors for the first observer and 5 cases for the second observer. Results of examined donors' liver biopsies revealed histopathological liver fat content $<5 \%$ (grade 0 ) in 40 donors and $5-10 \%$ (grade 1) in 7 donors (Table 2).

The estimated sensitivity and specificity for liver steatosis detection with MR fat fraction technique were $85.7 \%$ and $97.5 \%$, respectively, positive predictive value was found to be $85.7 \%$, negative predictive value was
97.5\% with a cutoff of point 5.6 with area under the curve 0.98 at $95 \%$ confidence interval of 0.98 to 1.01 , accuracy of $95.7 \%$ (Figs. 2 and 3). There was an excellent inter-reader agreement between both readers in the estimation of MR liver fat fraction ( $r=0.969$ at $95 \%$ confidence interval of 0.946 to 0.983$)$.

\section{Discussion}

Living donor liver transplantation is one of the present treatment options of end-stage liver diseases. Living donor safety is a remarkable priority in such treatment modality. Hepatic steatosis is a serious risk factor in liver transplantation. A fatty liver of donor is associated with increased morbidity in both donor and recipient $[1,18,19]$. It was stated that hepatic steatosis of $\geq 10 \%$ is one of most common causes for

Table 2 Median and range of estimated MR and histopathological hepatic fat fractions and grades

\begin{tabular}{lll}
\hline & $n=47$ & Percent \\
\hline $\begin{array}{ll}\text { MR Fat \% (1st observer) } \\
\text { Median(min-max) }\end{array}$ & $2.5(1.1-16.0)$ & \\
$\begin{array}{l}\text { MR fat \% (2nd observer) } \\
\text { Median (min-max) }\end{array}$ & $3.26(1.43-14.7)$ & \\
MR Grade (1st observer) & & \\
0 & 43 & 91.5 \\
1 & 4 & 8.5 \\
MR Grade (2nd observer) & & \\
0 & 42 & 89.4 \\
1 & 5 & 10.6 \\
Pathological fat grade & & 85.1 \\
0 & 40 & 14.9 \\
1 & 7 & \\
\hline
\end{tabular}




\section{ROC Curve}

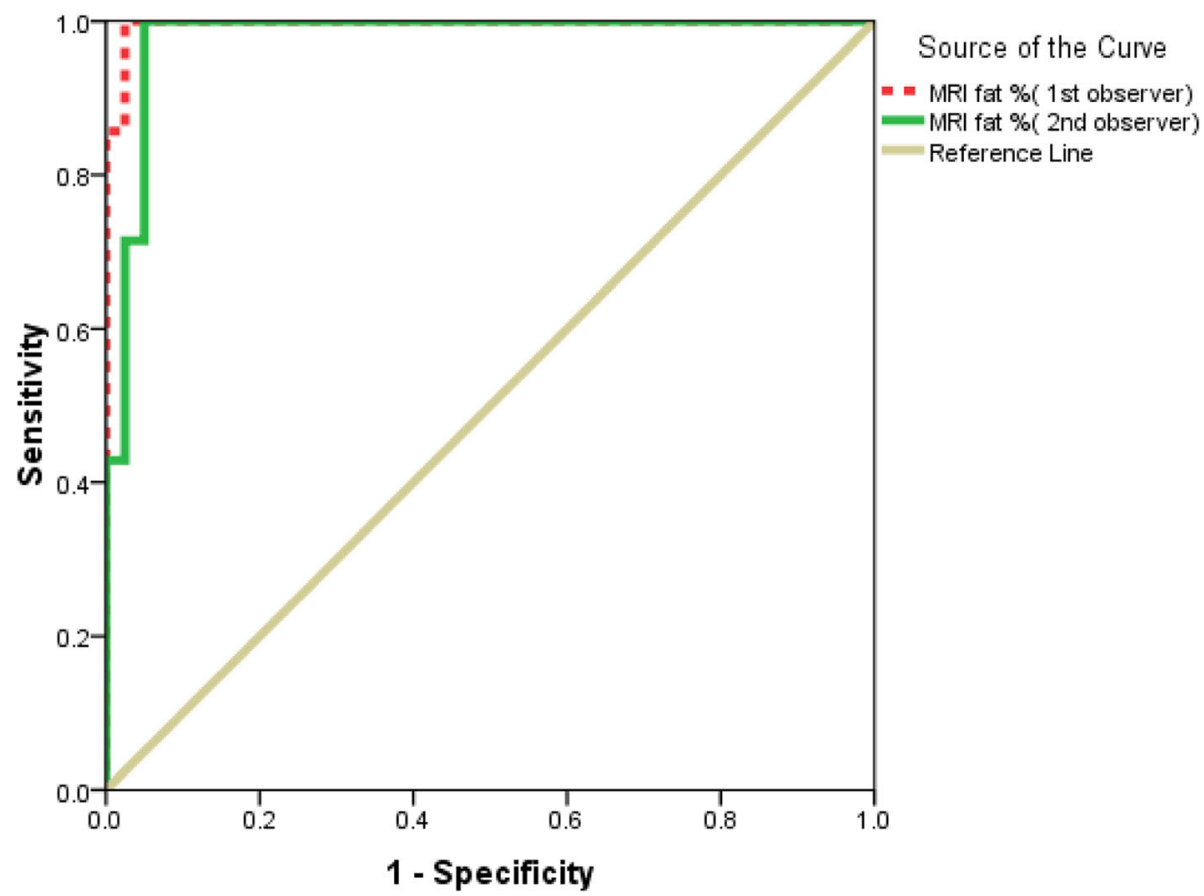

Fig. 2 ROC curve demonstrating the diagnostic accuracy of MR hepatic proton density fat fraction technique in estimation of hepatic steatosis

donor disqualification [6]. Therefore, accurate quantification of liver fat content is essential for assessment of the eligibility and safety of potential living donors.

There have been many attempts for noninvasive evaluation hepatic steatosis by using different imaging modalities $[9,11,20]$. MR techniques can decompose fat and water signal of the liver, enabling more accurate assessment of the amount of fat in the liver than other imaging modalities [5].
Results of this study revealed that MR hepatic PDFF using IDEAL technique proved to be a valid method for quantitative evaluation of hepatic steatosis in comparison to histopathology. This kept up with many recent previous studies who stated that this supports the potential use of MR PDFF technique as a reliable noninvasive quantitative method for assessment of hepatic steatosis $[1,5,10,21]$.

Other MR techniques have been previously used for assessment of hepatic steatosis such as hydrogen MR

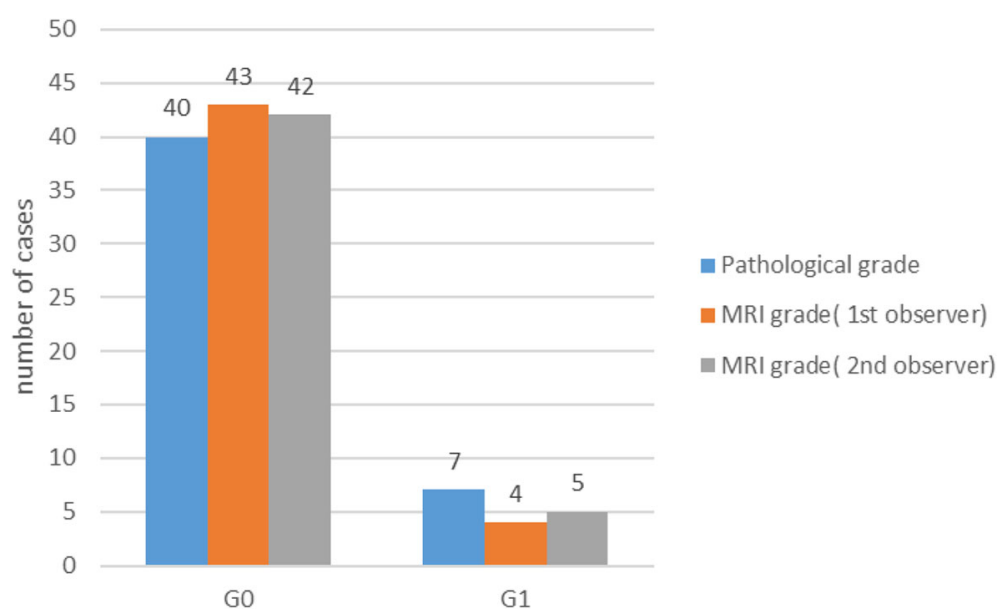

Fig. 3 Bar chart for frequency of MR hepatic fat fraction grades versus histopathological grades of hepatic steatosis 
spectroscopy. MR HFF technique and MR spectroscopy were declared to be similar in sensitivity and specificity. Despite its validity, MR spectroscopy needs a long acquisition time and sophisticated post-processing and data analysis [5, 22].

In this study, we were satisfied to use MR hepatic PDFF technique as it is easier, simpler, having less time, and can measure fat in any part of the liver, and this choice was in agreement with Bonekamp et al., Awai et al., and Harald et al. who demonstrated that MRIPDFF technique has a strong correlation with MR spectroscopy $\left(r^{2}=0.98\right)[2,10,23]$.

Multiple-segment hepatic fat fraction measurements can be obtained using IDEAL sequence for proper quantification of hepatic fat, thus reducing sampling error compared to MR spectroscopy which is usually carried out with single voxel technique for assessment of hepatic steatosis and even liver biopsy $[1,5,8]$.

The major strengths of this study include the use of adult subjects with liver biopsy-confirmed percentage and grade of hepatic steatosis. The study utilized a wellvalidated, simple MR PDFF technique (IDEAL sequence) which has overcome drawbacks of previous used MR fat assessment techniques by correcting for $\mathrm{T} 1$ bias, $\mathrm{T} 2$ * decay, and the spectral complexity of fat. Moreover, this technique corrects all of the known confounding factors that interfere with the measurement of hepatic fat fraction with MRI [5]. In addition, all patients underwent the same liver fat mapping with assessment of multiple liver segments. Also, the time interval between biopsy and MRI was short (average 1 month).

However, this study had many limitations, such as relatively small number of donors and using a 1.5-Tesla MR scanner, not higher MR scanner for examination. Lower magnetic field strength systems decrease the chemical shift effect, reducing the separation of fat and water MR signals. However, Kang et al. and Middleton et al. declared that no difference of calculated PDFFs using 1.5-T and 3-T MR scanners showed no difference in their results $[24,25]$.

\section{Conclusion}

In conclusion, noninvasive MR hepatic PDFF technique with IDEAL sequence is a precise reliable technique for quantitative assessment of hepatic steatosis with high sensitivity (85.7\%), specificity (97.5\%), and accuracy of (95.7\%). It is recommended to be a part of preoperative liver evaluation in living donors and selectively performing liver biopsy in donor candidates diagnosed to have substantial steatosis based on MR PDFF findings.

Ethics approval and consent for participation This study was approved by the Institutional Research Board of Mansoura Faculty of Medicine at Mansoura University in Egypt, reference number of approval R.19.09.632. Informed consent was waived because this was a retrospective study.

\section{Abbreviations}

MR: Magnetic resonance; PDFF: Proton density fat fraction; IDEAL: Iterative decomposition of water and fat; US: Ultrasound; MRI: Magnetic resonance imaging; 1H: Hydrogen 1; SD: Standard deviation; 3D: Three-dimensional; TR: Repetition time; TE: Echo time; NEX: Number of excitation; FOV: Field of view; GRE: Gradient echo; ROI: Region of interest; ROC: Receiver operating characteristic; T: Tesla

\section{Acknowledgements}

Not applicable.

\section{Authors' contributions}

FMS prepared MRI cases, performed MRI data analysis and statistical analysis, prepared figures and tables, and wrote and revised the manuscript. SAM suggested and developed the research idea, reviewed literature, performed MRI data analysis, and revised the manuscript. RMA collected and analyzed data, reviewed literature, and helped in manuscript editing. MAW developed the research idea, reviewing literature, data collection, and analysis and revised the manuscript. All authors read and approved the final manuscript.

\section{Funding}

This study had no funding from any resource.

Availability of data and materials

The datasets used and analyzed during the current study are available from the corresponding author on reasonable request.

Consent for publication

Not applicable.

\section{Competing interests}

The authors declare that they have no competing interests.

\section{Author details}

${ }^{1}$ Radiology Department, Faculty of Medicine, Mansoura University, Mansoura, Egypt. ${ }^{2}$ Gastroenterology Surgery Center, Faculty of Medicine, Mansoura University, Mansoura, Egypt.

Received: 26 September 2019 Accepted: 1 October 2019 Published online: 26 November 2019

\section{References}

1. Chiang H, Lin L, Li C et al. (2014) Magnetic resonance fat quantification in living donor liver transplantation. Transplantation Proceedings; 46: 666-668.

2. Bonekamp S, Tang A, Mashhood A et al (2014) Spatial distribution of MRI-determined hepatic proton density fat fraction in adults with nonalcoholic fatty liver disease. J Magn Reson Imaging 39(6):1525-1532

3. Yki-Järvinen $H$ (2016) Diagnosis of non-alcoholic fatty liver disease (NAFLD). Diabetologia 59:1104-1111

4. Shin YH, Ko JS, Kim GS et al (2012) Impact of hepatic macrovesicular and microvesicular steatosis on the postoperative liver functions after right hepatectomy in living donors. Transplant Proc 44(2):512-515

5. Hwang I, Lee J, Lee K et al (2014) Hepatic steatosis in living liver donor candidates: preoperative assessment by using breath-hold triple-echo MR imaging and 1H MR spectroscopy. Radiology 271(3):730-738

6. Sharma A, Ashworth A, Behnke M, Cotterell A, Posner M, Fisher RA (2013) Donor selection for adult-to-adult living donor liver transplantation: well begun is half done. Transplantation 95(3):501-506

7. El-Badry AM, Breitenstein S, Jochum W et al (2009) Assessment of hepatic steatosis by expert pathologists: the end of a gold standard. Ann Surg 250(5):691-697

8. Choi Y, Lee J, Yi N et al (2015) Heterogeneous living donor hepatic fat distribution on MRI chemical shift imaging. Annals of Surgical Treatment and Research 89(1):37-42

9. Lee SW, Park SH, Kim KW et al (2007) Unenhanced CT for assessment of macrovesicular hepatic steatosis in living liver donors: comparison of visual grading with liver attenuation index. Radiology 244(2):479-485

10. Awai H, Newton K, Sirlin C, Behling C, Schwimmer J (2014) Evidence and recommendations for imaging liver fat in children, based upon systematic review. Clin Gastroenterol Hepatol. 12(5):765-773 
11. Reeder SB, Cruite I, Hamilton G, Sirlin CB (2011) Quantitative assessment of liver fat with magnetic resonance imaging and spectroscopy. J Magn Reson Imaging 34(4):729-749

12. Paparo F, Cenderello G, Revelli M et al (2015) Diagnostic value of MRI proton density fat fraction for assessing liver steatosis in chronic viral C hepatitis. BioMed Research International Volume, Article ID 758164:1-11

13. Noureddin M, Lam J, Peterson M et al (2013) Utility of magnetic resonance imaging versus histology for quantifying changes in liver fat in nonalcoholic fatty liver disease trials. Hepatology 58(6):1930-1940

14. Idilman I, Aniktar H, Idilman R et al (2013) Hepatic steatosis quantification by proton density fat fraction with MR imaging versus liver biopsy. Radiology 267(3):767-775

15. Kinner S, Reeder S, Yokoo T (2016) Quantitative imaging biomarkers of NAFLD. Dig Dis Sci. 61(5):1337-1347

16. Qayyum A, Nystrom M, Noworolski S et al (2012) MRI steatosis grading: development and initial validation of a color mapping system. Gastrointestinal imaging 198:582-588

17. Zhang H, Fu J, Lai C et al (2018) Feasibility of balanced steady state free precession sequence at $1.5 \mathrm{~T}$ for the evaluation of hepatic steatosis in obese children and adolescents. European Radiology 28:4479-4487

18. Wieckowska A, McCullough AJ, Feldstein AE (2007) Noninvasive diagnosis and monitoring of nonalcoholic steatohepatitis: present and future. Hepatology 46:582-589

19. Schwimmer JB, Deutsch R, Kahen T, Lavine JE, Stanley C, Behling C (2006) Prevalence of fatty liver in children and adolescents. Pediatrics 118:1388-1393

20. Kim SH, Lee JM, Kim JH et al (2005) Appropriateness of a donor liver with respect to macrosteatosis: application of artificial neural networks to US images-initial experience. Radiology 234(3):793-803

21. Permutt Z, Lee T, Peterson M, Seki E, Brenner D, Sirlin C, Loomba R (2012) Correlation between liver histology and novel magnetic resonance imaging in adult patients with non-alcoholic fatty liver disease - MRI accurately quantifies hepatic steatosis in NAFLD. Aliment Pharmacol Ther 36:22-29

22. Koplay M, Sivri M, Erdogan H, Nayman A (2015) Importance of imaging and recent developments in diagnosis of nonalcoholic fatty liver diseases. World J Hepatol April 18 7(5):769-776

23. Harald K, Perry P, Mark K et al (2017) Accuracy of liver fat quantification with advanced $\mathrm{CT}$, MRl, and ultrasound techniques: prospective comparison with MR spectroscopy. American journal of Roentgenology 208:92-100

24. Kang GH, Cruite I, Shiehmorteza M et al (2011) Reproducibility of MRI-determined proton density fat fraction across two different MR scanner platforms. J Magn Reson Imaging 34(4):928-934

25. Middleton $\mathrm{M}$, Elhamy $\mathrm{H}$, Hooker $\mathrm{C}$ et al (2017) Agreement between magnetic resonance imaging proton density fat fraction measurements and pathologist-assigned steatosis grades of liver biopsies from adults with nonalcoholic steatohepatitis. Gastroenterology 153:753-761

\section{Publisher's Note}

Springer Nature remains neutral with regard to jurisdictional claims in published maps and institutional affiliations.

\section{Submit your manuscript to a SpringerOpen ${ }^{\circ}$ journal and benefit from:}

- Convenient online submission

- Rigorous peer review

- Open access: articles freely available online

- High visibility within the field

- Retaining the copyright to your article

Submit your next manuscript at $\boldsymbol{\nabla}$ springeropen.com 